\title{
NEOWISE OBSERVATIONS OF COMET C/2013 A1 (SIDING SPRING) AS IT APPROACHES MARS
}

\author{
R. Stevenson ${ }^{1}$, J. M. BAuer ${ }^{1,2}$, R. M. Cutri ${ }^{2}$, A. K. Mainzer ${ }^{1}$, And F. J. Masci ${ }^{2}$ \\ ${ }^{1}$ Jet Propulsion Laboratory, California Institute of Technology, 4800 Oak Grove Drive, Pasadena, CA 91109, USA \\ ${ }^{2}$ Infrared Processing and Analysis Center, California Institute of Technology, Pasadena, CA 91125, USA \\ Received 2014 October 14; accepted 2014 December 4; published 2014 December 29
}

\begin{abstract}
The Near-Earth Object Wide-field Infrared Survey Explorer mission observed comet C/2013 A1 (Siding Spring) three times at $3.4 \mu \mathrm{m}$ and $4.6 \mu \mathrm{m}$ as the comet approached Mars in 2014. The comet is an extremely interesting target since its close approach to Mars in late 2014 will be observed by various spacecraft in situ. The observations were taken in 2014 January, July, and September when the comet was at heliocentric distances of 3.82 AU, 1.88 AU, and $1.48 \mathrm{AU}$. The level of activity increased significantly between the January and July visits but then decreased by the time of the observations in September, approximately four weeks prior to its close approach to Mars. In this work, we calculate Af $\rho$ values and $\mathrm{CO} / \mathrm{CO}_{2}$ production rates.
\end{abstract}

Key word: comets: individual (C/2013 A1 (Siding Spring))

\section{INTRODUCTION}

Comet C/2013 A1 (Siding Spring) presents both risk and opportunity at its close approach to Mars on 2014 October 19. It will pass approximately $135,000 \mathrm{~km} \pm 5000 \mathrm{~km}$ from the planet's center (Farnocchia et al. 2014)—close enough for detailed observations by spacecraft at Mars but also close enough for ejected dust and gas to reach Mars' atmosphere, potentially affecting spacecraft in orbit. The potential risk posed by the dust has been downgraded through careful dynamical modeling. Early studies suggested relatively high amounts of dust would reach the Martian atmosphere (Ye \& Hui 2014; Moorhead et al. 2014). Later studies that included lower ejection velocities and radiation pressure effects showed that only a low fluence of old, larger dust grains is expected to reach the atmosphere (Tricarico et al. 2014; Kelley et al. 2014). However, it is possible that the gas coma may sufficiently excite the atmosphere of Mars to cause increased drag on orbiting satellites (Yelle et al. 2014). The comet is also interesting in its own right as a long-period comet on a near-parabolic retrograde orbit, bringing primitive material in from the Oort Cloud.

In this Letter, we use data from the Near-Earth Object Wide-Field Infrared Survey Explorer (NEOWISE) reactivation (Mainzer et al. 2014) mission to characterize the comet's activity on three occasions in 2014 January, July, and September as the comet approached Mars. We derive dust and gas production rates using near-infrared wide-field images and examine the evolving morphology of the coma.

\section{DATA ACQUISITION AND REDUCTION}

The NEOWISE mission utilizes the Wide-Field Infrared Survey Explorer (WISE) spacecraft (Wright et al. 2010), which completed an all-sky survey at four wavelengths of 3.4, 4.6, 12, and $22 \mu \mathrm{m}$ from 2010 January to August using a $40 \mathrm{~cm}$ cryogenically cooled telescope. After the depletion of the spacecraft's solid hydrogen, the mission could still operate at the two shortest wavelengths of 3.6 and $4.6 \mu \mathrm{m}$. This phase of the mission was renamed NEOWISE (Mainzer et al. 2011) and continued for several months until 2011 February 1 after which the spacecraft was placed into hibernation. The spacecraft was then reactivated in 2013 December for the start of the NEOWISE reactivation mission (Mainzer et al. 2014). The telescope is conducting an all-sky survey in a low-Earth orbit, taking images of the sky every $11 \mathrm{~s}$ at a solar elongation of $\sim 92.5$. The images have an effective exposure time of $7.7 \mathrm{~s}$ and an effective pointsource FWHM of $6{ }^{\prime \prime} 25$. There is approximately $10 \%$ overlap between successive frames, allowing for the linking of moving object detections. The images, which are $47^{\prime}$ on a side, undergo instrumental and astrometric calibration, followed by source extraction at the Infrared Processing and Analysis Center (IPAC) at the California Institute of Technology (Wright et al. 2010). All data from the WISE prime mission in 2010 have been publicly released via NASA's Infrared Science Archive (IRSA), ${ }^{3}$ and the first data release from the NEOWISE reactivation mission will be in 2015 March through IRSA.

The motion of comet Siding Spring meant that it was observed by NEOWISE on three separate occasions, hereafter referred to as "visits," on 2014 January 16-17, July 28-29, and September 21-22. Observing details are given in Table 1.

We used predicted positions from the Jet Propulsion Laboratory's Horizons service ${ }^{4}$ and the Moving Object Search Tool ${ }^{5}$ (Cutri et al. 2012) to identify the images covering comet Siding Spring. Trailing during each $7.7 \mathrm{~s}$ exposure was at most lower than $1^{\prime \prime}$, which is significantly less than the FWHM. We coadded the images of the comet in the moving reference frame for each visit using the ICORE image co-addition package that includes outlier rejection (Masci 2013). This improved the signalto-noise ratio and resampled the pixel scale from 2.75 pixel $^{-1}$ to $1^{\prime \prime} .0$ pixel $^{-1}$. The coadded images were centered on the predicted position of the comet. Two-band color images of comet Siding Spring from each visit are shown in Figure 1. The comet appeared active at each visit.

We used circular apertures with radii of $11^{\prime \prime}$ to perform photometry on each coadded image. Counts were converted to fluxes using instrumental zero points using the same method as used for WISE catalog source data (Wright et al. 2010). The background was estimated and subtracted using the modal value in an aperture of at least $1.4 \operatorname{arcmin}^{2}$ located $\geqslant 1^{\prime}$ from the nucleus and far from the coma. The dominant source of error is the uncertainty on the absolute calibration. We fit a reflected light model to the observed data at $3.4 \mu \mathrm{m}$, making the assumption that the flux observed is due to reflected light from dust grains and that the grains have a neutral reflectance. The reflected light is thus

\footnotetext{
3 http://irsa.ipac.caltech.edu/

4 http://ssd.jpl.nasa.gov/horizons.cgi

5 http://irsa.ipac.caltech.edu/applications/MOST/
} 

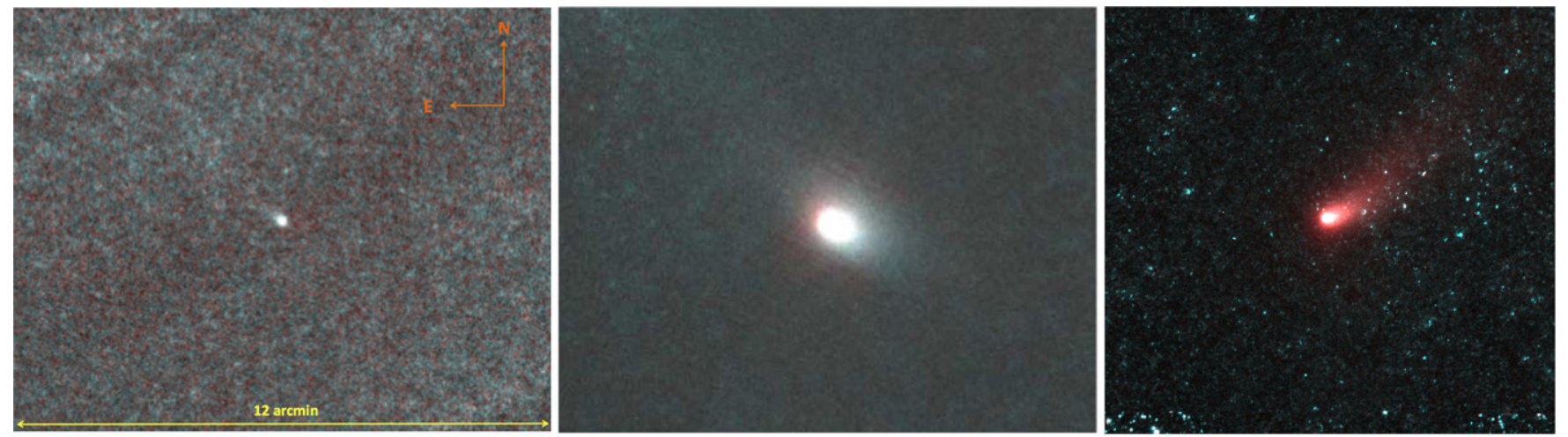

Figure 1. Two-band false-color images of Comet C/2013 A1 (Siding Spring) as observed by the NEOWISE mission in 2014 January (left panel), July (middle panel), and September (right panel). The $3.4 \mu \mathrm{m}$ band is shown as blue, while the $4.6 \mu \mathrm{m}$ band is shown in red. The contrast and stretch of each image is adjusted for clear viewing and are not constant across the panels. However, the scale and orientations, as indicated in the left panel, are the same for all images.
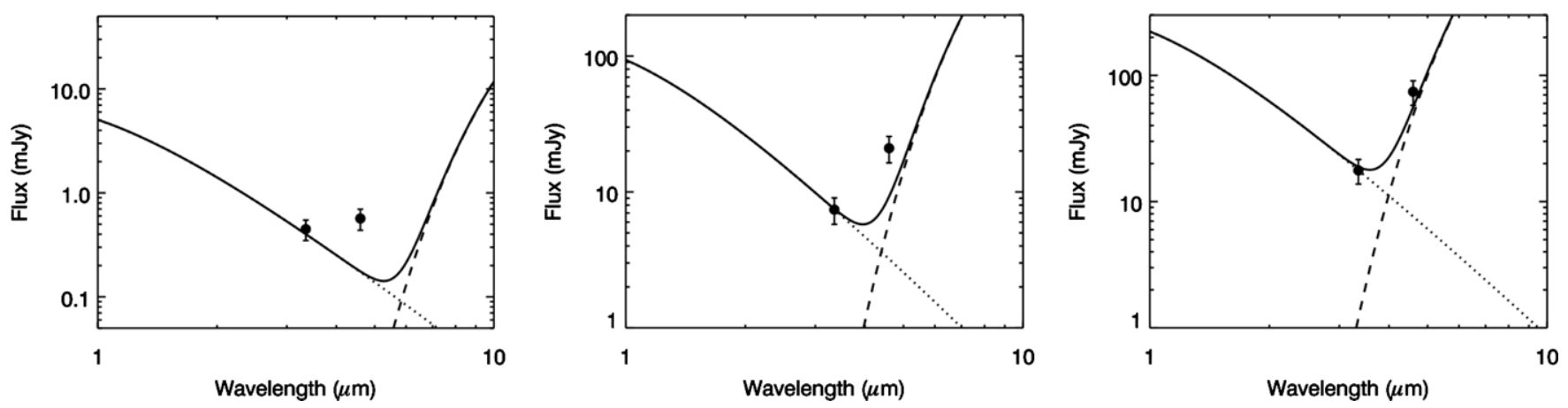

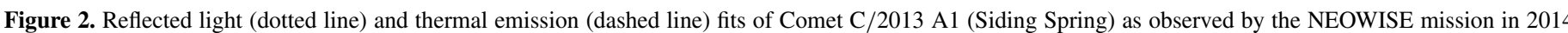

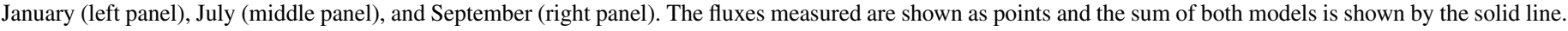

Table 1

Observations

\begin{tabular}{|c|c|c|c|}
\hline Quantity & January Visit & July Visit & September Visit \\
\hline Date $(\mathrm{UT})^{\mathrm{a}}$ & 2014 Jan 16.62 & $2014 \mathrm{Jul} 28.87$ & 2014 Sep 21.73 \\
\hline $\mathrm{N}^{\mathrm{b}}$ & 16 & 11 & 9 \\
\hline$\Delta^{\mathrm{c}}(\mathrm{AU})$ & 3.67 & 1.54 & 1.04 \\
\hline $\mathrm{r}_{H}{ }^{\mathrm{d}}(\mathrm{AU})$ & 3.82 & 1.88 & 1.48 \\
\hline$\alpha^{\mathrm{e}}\left({ }^{\circ}\right)$ & 14.9 & 32.7 & 42.6 \\
\hline Image scale $\left(\mathrm{km} \mathrm{arcsec}^{-1}\right)$ & 2662 & 1117 & 754 \\
\hline $3.4 \mu \mathrm{m}$ flux (mJy) & $0.5 \pm 0.1$ & $7.4 \pm 1.6$ & $18 \pm 4$ \\
\hline $4.6 \mu \mathrm{m}$ flux (mJy) & $0.6 \pm 0.1$ & $21 \pm 5$ & $74 \pm 16$ \\
\hline $\operatorname{Af} \rho(\mathrm{cm})$ & $432 \pm 21$ & $726 \pm 40$ & $724 \pm 40$ \\
\hline $\mathrm{CO}\left(\right.$ molecules s $\left.^{-1}\right)$ & $(3.56 \pm 0.36) \times 10^{27}$ & $(1.65 \pm 0.17) \times 10^{28}$ & $(1.25 \pm 0.13) \times 10^{28}$ \\
\hline $\mathrm{CO}_{2}\left(\right.$ molecules s $\left.^{-1}\right)$ & $(3.42 \pm 0.34) \times 10^{26}$ & $(1.56 \pm 0.16) \times 10^{27}$ & $(1.18 \pm 0.12) \times 10^{27}$ \\
\hline
\end{tabular}

Notes.

a Mid-point of median stack.

${ }^{\mathrm{b}}$ Number of individual images used.

${ }^{\mathrm{c}}$ Geocentric distance at mid-point.

${ }^{\mathrm{d}}$ Heliocentric distance at mid-point.

${ }^{\mathrm{e}}$ Phase angle at mid-point.

effectively the solar flux scaled to the $3.4 \mu \mathrm{m}$ signal. We neglected light reflected or emitted by the nucleus, the size of which is unknown at this time because it has been obscured by dust since discovery. If the nucleus is on the order of $1 \mathrm{~km}$ in radius, it would contribute a few tenths of a $\mu \mathrm{Jy}$ to the fluxes observed during the 2014 January visit when the comet was least active and the nucleus likely contributed the largest fraction of the light. This is significantly less than the uncertainty in the flux measurements and we conclude that the nucleus contributes a negligible amount of light. We also computed a theoretical thermal contribution to the flux by calculating the blackbody radiation that would be emitted by the quantity of dust estimated from the $3.4 \mu \mathrm{m}$ flux. We used a Planck function and assumed an emissivity $\sim 0.9$, so that the temperature of the grains scaled as $286 \mathrm{~K} \times r_{H}^{-1 / 2}$. It is entirely possible that the grain spectral energy distribution is more complicated, but the singular data point at $3.4 \mu \mathrm{m}$ only allows for a simplified dust model constraint. The measured fluxes for each visit are listed in Table 1 and shown in Figure 2 with the reflected light and predicted thermal light models. 


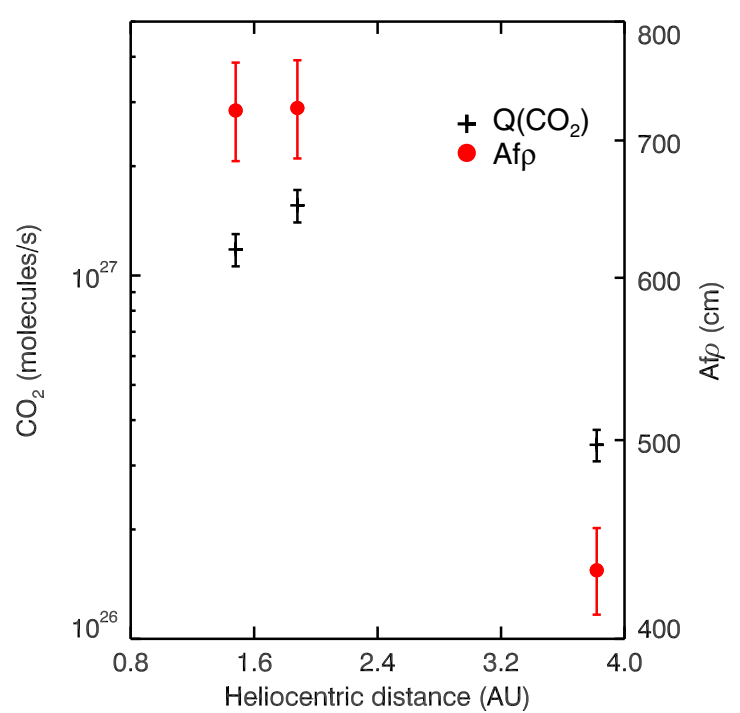

Figure 3. Variation of $\operatorname{Af} \rho$ and $\mathrm{CO}_{2}$ production over time as observed by NEOWISE.

\section{RESULTS}

\subsection{Dust Production}

We converted the flux measured at $3.4 \mu \mathrm{m}$ to the quantity Af $\rho$ - a proxy for the dust production rate discussed in A'Hearn et al. (1984)—using Equation (1).

$$
A f \rho=\frac{\left(2 \Delta r_{H}\right)^{2}}{\rho} \frac{F_{\text {comet }}}{F_{\odot}},
$$

where $A$ is the Bond albedo of the dust at the phase angle of observation, $f$ is the filling factor of the dust grains within the aperture, $\rho$ is the aperture size in centimeters, $\Delta$ and $r_{H}$ are the geocentric and heliocentric distances in centimeters and $\mathrm{AU}$, respectively, and $F_{\text {comet }}$ and $F_{\odot}$ are the flux from the comet and the Solar flux at $1 \mathrm{AU}$, respectively. As before, we used $11^{\prime \prime}$ radius apertures. We estimate the uncertainties on our measurements by examining the spread of results obtained when using apertures of radii $9^{\prime \prime}, 11^{\prime \prime}$, and $22^{\prime \prime}$ since, ideally, the quantity calculated should be independent of chosen aperture size. However, it is quite possible that the spread in these values may in part be due to non-idealized behavior, i.e., that the coma flux does not drop off as $\rho^{-1}$. Residuals from the removal of inertial background sources from the co-added image may contribute to the $A f \rho$ uncertainty as well. We measure $A f \rho$ as $432 \pm 21 \mathrm{~cm}$ during the 2014 January visit, $726 \pm 40 \mathrm{~cm}$ during the July visit, and $724 \pm 40 \mathrm{~cm}$ during the September visit. These $A f \rho$ values are provided for comparison with each other. The values are not corrected for phase angle effects since these effects are poorly constrained for infrared wavelengths, nor do the uncertainties include the component from the uncertainty in the absolute photometric calibration. We report these values in Table 1 and show how $A f \rho$ varied with heliocentric distance in Figure 3. The $A f \rho$ value reported by Bodewits et al. (2014) from observations in late May falls between our Af $\rho$ values when using the same phase-angle correction method. ${ }^{6}$ Because the phase-angle correction at $3.4 \mu \mathrm{m}$ is not well-constrained for comet dust, we hesitate to make any further direct comparisons between the optical and infrared $A f \rho$ values when they are not simultaneous.

\footnotetext{
6 http://asteroid.lowell.edu/comet/dustphase.html
}

\subsection{Anomalous Emission at $4.6 \mu \mathrm{m}$}

At $4.6 \mu \mathrm{m}$ the observed flux exceeds that predicted by the combined reflected and thermal light models. In 2014 January we find that the excess emission is at the $3 \sigma$ level, while it drops to $2.5 \sigma$ and $1.1 \sigma$ in the July and September visits, respectively. We note that the uncertainties are dominated by systematics that correlate with the signal at $3.4 \mu \mathrm{m}$ and thus the uncertainties at $4.6 \mu \mathrm{m}$ are likely overestimated. The September band-excess detection is notably weak. However, the $3.4 \mu \mathrm{m}$ and $4.6 \mu \mathrm{m}$ relative photometry are better constrained, since the relative uncertainties are $\sim 7 \%$ (Cutri et al. 2012), considerably less than the uncertainties listed in Table 1. The NEOWISE bandpass at $4.6 \mu \mathrm{m}$ contains emission features from $\mathrm{CO}_{2}\left(\nu_{3}\right.$ band) at $4.26 \mu \mathrm{m}$ and $\mathrm{CO}(v=1-0$ band $)$ at $4.67 \mu \mathrm{m}$. Both species have sufficiently long photodissociation lifetimes to be responsible for the observed excess flux. We therefore interpret the excess emission as being due to optically thin gaseous emission and convert the excess flux, $F$, to an average column density, $\langle N\rangle$, using Equation (2):

$$
\langle N\rangle=F 4 \pi \Delta^{2} \frac{\lambda}{h c} \frac{r_{h}^{2}}{g} \frac{1}{\pi \rho^{2}},
$$

where the symbols have the same meanings as in Equation (1), $\lambda$ is the wavelength of observation, $h$ is Planck's constant, $c$ is the speed of light, and $g$ is the fluorescence efficiency for the chosen gas species. $F$ is the excess flux density in the $4.6 \mu \mathrm{m}$ bandpass and is integrated over the $\mathrm{CO} / \mathrm{CO}_{2}$ band, after the inband dust signal contribution has been removed, as described in Pittichová et al. (2008). We are unable to distinguish between $\mathrm{CO}$ and $\mathrm{CO}_{2}$ emission with just the NEOWISE data since the bandpass spans both features and therefore present two scenarios in Table 1 where $100 \%$ of the excess flux is due to $\mathrm{CO}$ emission or it is entirely due to $\mathrm{CO}_{2}$ emission. We assume fluorescence efficiencies at a heliocentric distance of 1 AU for the $\mathrm{CO} v=1-0$ and the $\mathrm{CO}_{2} v_{3}$ bands of $2.46 \times 10^{-4} \mathrm{~s}^{-1}$ and $2.86 \times 10^{-3} \mathrm{~s}^{-1}$, respectively (Crovisier \& Encrenaz 1983). We then use the average column densities to calculate the production rates, $Q$ in molecules $\mathrm{s}^{-1}$, using Equation (3):

$$
Q=\langle N\rangle 2 \rho v \times 10^{5},
$$

in which $\langle N\rangle$ and $\rho$ have their previous definitions, $v$ is the ejection velocity of the gas in $\mathrm{km} \mathrm{s}^{-1}$, and $10^{5}$ is a conversion factor (Pittichová et al. 2008). We take the ejection velocity to be $0.6 \mathrm{~km} \mathrm{~s}^{-1}$ during the 2014 January visit when the comet was at a heliocentric distance of $3.82 \mathrm{AU}$ and assume that the velocity scales as $\sqrt{r_{H}}$ (Delsemme 1982). The production rates calculated are given in Table 1 and show that gas production increased overall by a factor of $\sim 3.5$ between 2014 January and September For these heliocentric distances, this kind of increase, proportional to $\sim r^{-1.8}$, is comparable to a $Q_{\mathrm{CO}_{2}} \sim r^{-2}$, possibly coinciding with a fixed source of constant area (Meech \& Svoren 2004). There was a decrease between the July visit and the September visit which may be attributable to a decrease in overall activity. Figure 3 shows the evolution of the $A f \rho$ quantity and $\mathrm{CO}_{2}$ production as the comet moves toward perihelion. There are comparatively few published $\mathrm{H}_{2} \mathrm{O}$ values for Siding Spring, but $Q_{\mathrm{H}_{2} \mathrm{O}}$ was measured to be $\sim 2 \times 10^{27}$ molecules $\mathrm{s}^{-1}$ when the comet was at $\sim 2.5 \mathrm{AU}$ (Bodewits et al. 2014). This would suggest a lower bound of $Q_{\mathrm{CO}_{2}} / Q_{\mathrm{H}_{2} \mathrm{O}}$ of $15 \%$ assuming water production increased with decreasing heliocentric distance from 3.8 to $2.5 \mathrm{AU}$. 


\section{CONCLUSIONS}

We have used data from the NEOWISE reactivation mission to monitor the activity of comet Siding Spring over nine months as it approaches Mars on a trajectory that will bring it within $\sim 135,000 \mathrm{~km}$ of the planet. Our conclusions from this work are as follows.

1. NEOWISE observed comet Siding Spring to be active from 2014 January to September. The quantity $A f \rho$ and $\mathrm{CO} / \mathrm{CO}_{2}$ production rates initially increased as the comet reached a heliocentric distance of $1.88 \mathrm{AU}$ but then decreased slightly even as the comet moved further inward.

2. The activity of the comet decreased between July and September, possibly due to depletion of volatile deposits on or near the surface.

3. The decrease in activity suggests the risk to assets at Mars was reduced since activity diminished as the comet approached Mars.

This publication makes use of data products from NEOWISE, which is a project of the Jet Propulsion Laboratory/California Institute of Technology, funded by the National Aeronautics and Space Administration. This research has made use of the NASA/IPAC Infrared Science Archive, which is operated by the Jet Propulsion Laboratory, California Institute of Technology, under contract with the National Aeronautics and Space Administration. R.S. gratefully acknowledges support from the NASA Postdoctoral Program.

\section{REFERENCES}

A'Hearn, M. F., Schleicher, D. G., Millis, R. L., Feldman, P. D., \& Thompson, D. T. 1984, AJ, 89, 579

Bodewits, D., Farnham, T., \& A'Hearn, M. F. 2014, CBET, 3888, 1

Crovisier, J., \& Encrenaz, T. 1983, A\&A, 126, 170

Cutri, R. M., Wright, E. L., Conrow, T., et al. 2012, Explanatory Supplement to the WISE All-Sky Data Release Products, 1

Delsemme, A. H. 1982, in IAU Colloq. 61, Comet Discoveries, Statistics, and Observational Selection (Cambridge: Cambridge Univ. Press), 85

Farnocchia, D., Chesley, S. R., Chodas, P. W., et al. 2014, ApJ, 790, 114

Kelley, M. S. P., Farnham, T. L., Bodewits, D., Tricarico, P., \& Farnocchia, D. 2014, ApJL, 792, L16

Mainzer, A., Bauer, J., Cutri, R. M., et al. 2014, ApJ, 792, 30

Mainzer, A., Bauer, J., Grav, T., et al. 2011, ApJ, 731, 53

Masci, F. 2013, ascl soft, 2010

Meech, K. J., \& Svoren, J. 2004, Using Cometary Activity to Trace the Physical and Chemical Evolution of Cometary Nuclei. Comets II 317

Moorhead, A. V., Wiegert, P. A., \& Cooke, W. J. 2014, Icar, 231, 13

Pittichová, J., Woodward, C. E., Kelley, M. S., \& Reach, W. T. 2008, AJ, 136, 1127

Tricarico, P., Samarasinha, N. H., Sykes, M. V., et al. 2014, ApJL, 787, L35

Wright, E. L., Eisenhardt, P. R. M., Mainzer, A. K., et al. 2010, AJ, 140, 1868

Ye, Q.-Z., \& Hui, M.-T. 2014, ApJ, 787, 115

Yelle, R. V., Mahieux, A., Morrison, S., Vuitton, V., \& Hörst, S. M. 2014, Icar, 237,202 